\title{
Design and Implementation of Medicine Dispenser
}

\author{
Rishit Dagli ${ }^{1}$ \\ Student, Thakur International School Member of Young Engineer's Club Science Kidz ${ }^{1}$
}

\begin{abstract}
Most of the people in the world face a problem of forgetting to take their medicines on time this device will help them to take their medicines on right time. It can also be used for deaf and blind people, it will also have a band with it which will have vibrator and LED (Light Emitting Diode) flasher as indicator so the user can be alerted about taking medicines. After this he has to go to the Medicine Dispenser and spread his hand. The device will detect the hand and according to the time will dispense the appropriate medicine. With some modifications this device can also be installed on streets and people can withdraw their required medicines, here a RFID (Radio Frequency Identification) card can be used to store the data and identify the person and validate it.
\end{abstract}

Keywords: Medicine Dispenser, Arduino Nano, Vibrator, Servo SG90, NRF 24L01, RTC DS3231, IR sensor, IOT, Node MCU

\section{INTRODUCTION}

Medicine Dispenser aims to help people to take their right medicine on right time. It will contain a band to be worn by the user, this band will contain a vibrator to alert the user that it is time for taking his medicine. It will also contain an NRF with which it can communicate with medicine vending machine and an RTC to maintain the date and time and show it to user too. The medicine vendor will have an IR sensor so if the user places his hand under the box he would get the right medicine according to the time. It will also have a conveyor belt through which the medicine can be dropped into the users hand, NRF to communicate with the watch and an internet module(Node MCU) which will enable it to use internet services and we can also input the time and name of medicines into the machine by an app or website, we can also detect the amount of medicine left in the dispenser , calculate the amount of medicine needed and according to the need order the medicines online through the internet module. To make this secure if the time has not arrived and someone puts his hands under the IR medicines will not be dispensed. This product mainly targets people who are old or blind or patients with memory problems but need to take medicines on daily basis. It will help them in not skipping their medicines.

This is the birds eye view of sensors and other equipment's used in the watch and vending machine

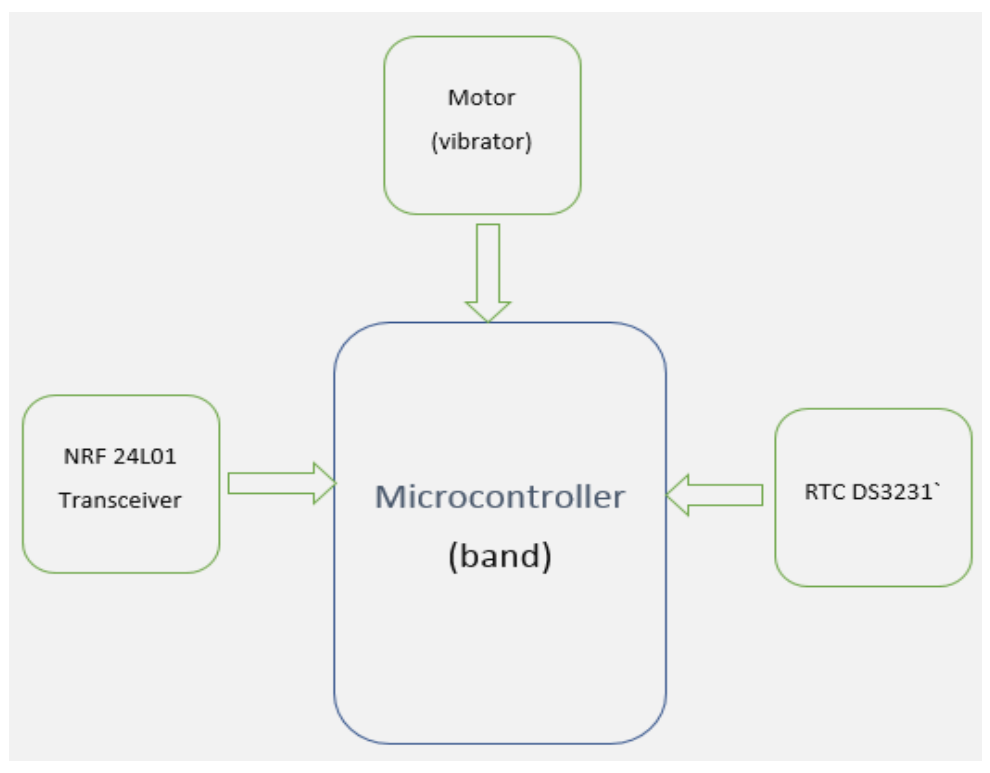




\section{International Advanced Research Journal in Science, Engineering and Technology}

Vol. 6, Issue 6, June 2019

This is the schematic diagram of my project

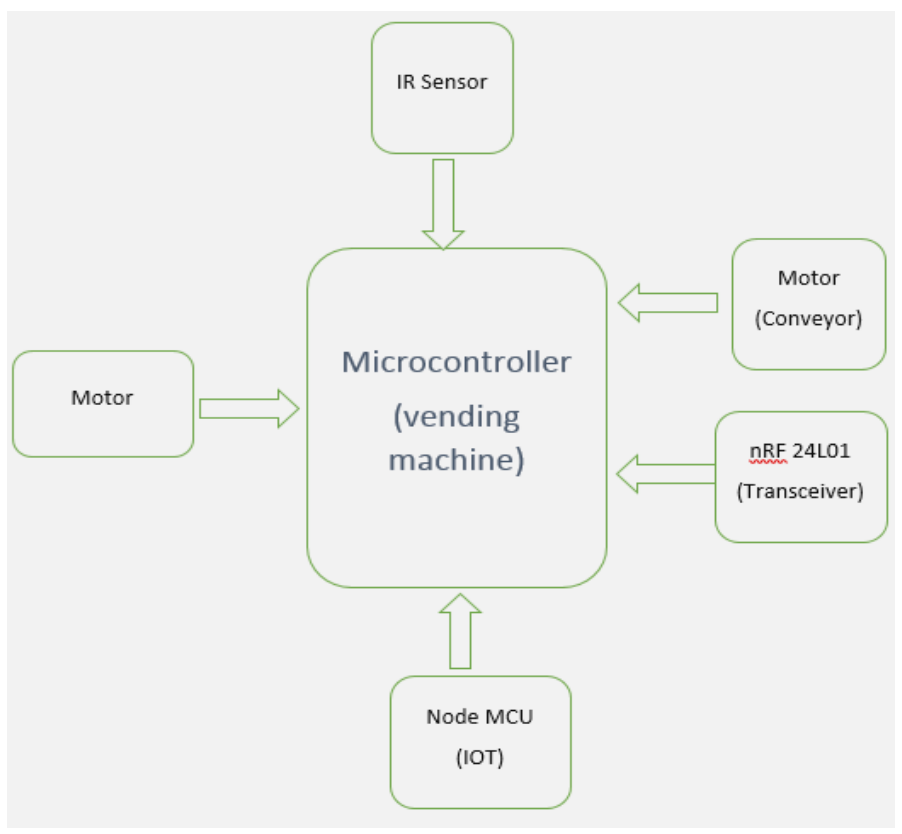

I have used the software fritzing to design the circuit diagrams and depicted the circuit diagram on Arduino Uno which can be used for prototyping but we can also use some other microcontroller like Raspberry Pi or ARM.

A. Band

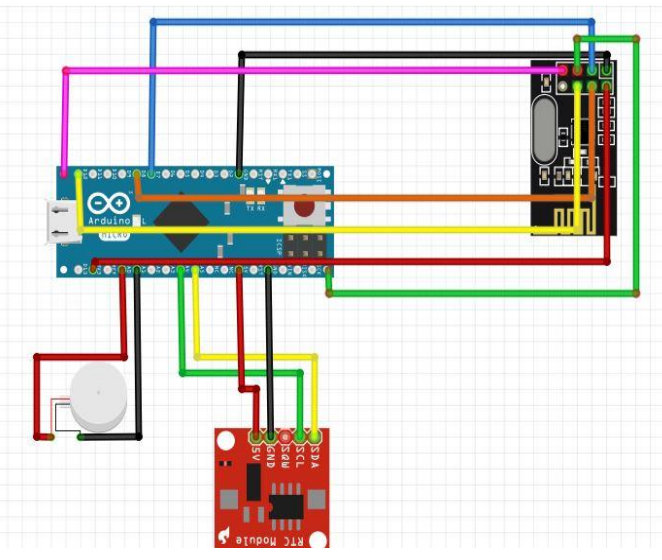

B. Vending Machine

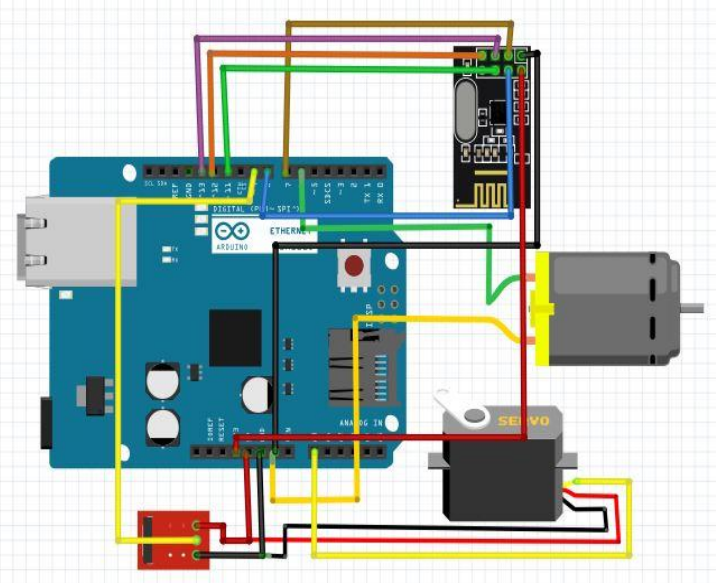




\title{
International Advanced Research Journal in Science, Engineering and Technology
}

\author{
Vol. 6, Issue 6, June 2019
}

The vending machine has an IOT platform like Node MCU or the ethernet shield. I have used an ethernet shield on top of Arduino Uno because of lower number of pins in Node MCU. Here the servo motor will be used to let medicines fall on the conveyor belt and the other motor will be used to run the conveyor belt.

\section{PROPOSED IMPLEMENTATIONS}

A microcontroller is a compact microcomputer designed to govern the operation of embedded systems in motor vehicles, robots, office machines, complex medical devices, mobile radio transceivers, vending machines, home appliances, and various other devices. A typical microcontroller includes a processor, memory and peripherals. This is an instance of Ardublock, it is a drag and drop application. This application makes it easy to write programs and debug errors. This is a program of NRF (Nordic Radio Frequency) receiver.

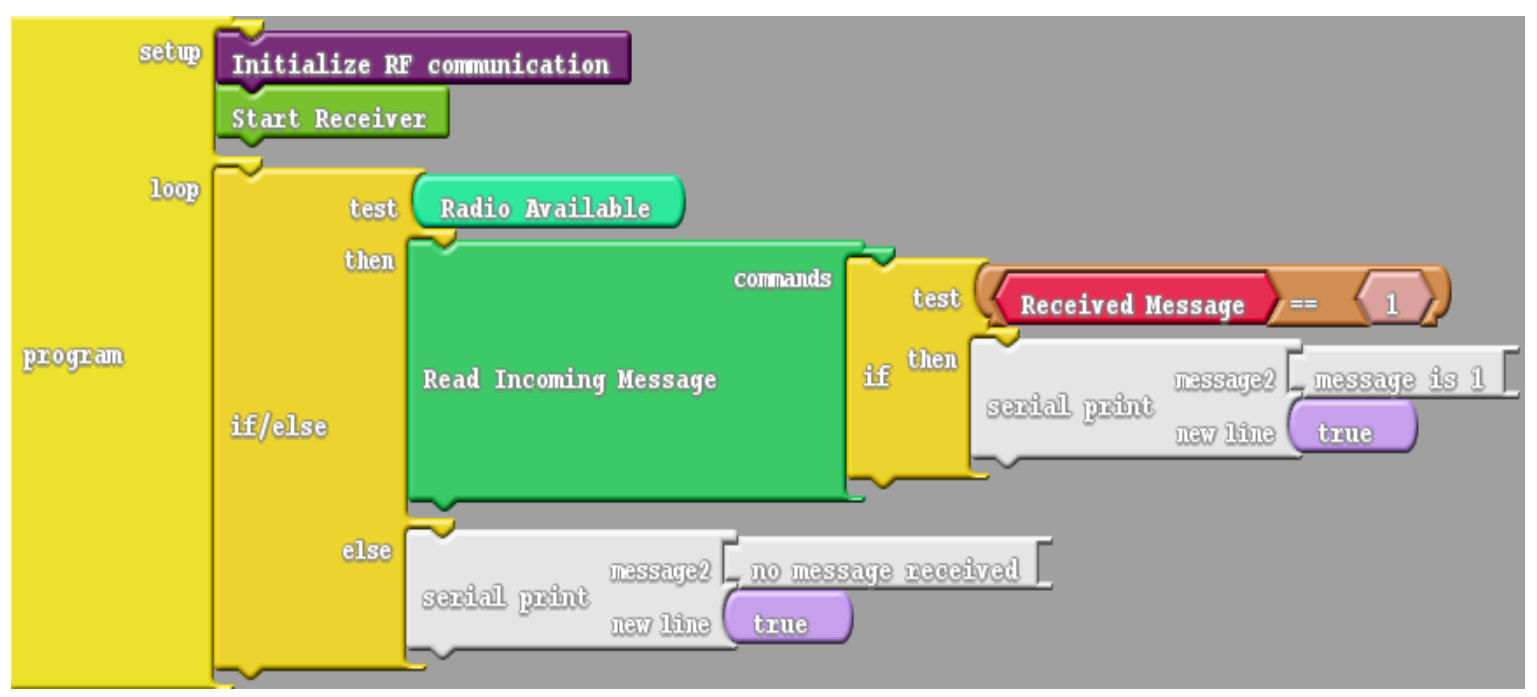

III. THEORY

\section{A. RTC}

The DS3231 is a low-cost, extremely accurate and fast $(400 \mathrm{kHz})$ I2C real-time clock (RTC) with an integrated temperature compensated crystal oscillator (TCXO) and crystal. The device incorporates a battery input, and maintains accurate timekeeping when main power to the device is interrupted. The RTC maintains seconds, minutes, hours, day, date, month, and year information. The date at the end of the month is automatically adjusted for months with fewer than 31 days, including corrections for leap year. The clock operates in either the 24-hour or 12-hour format with an $\mathrm{AM} / \mathrm{PM}$ indicator. Address and data are transferred serially through an I2C bidirectional bus.it can also give SquareWave Output Signal.

Applications

- $\quad$ Servers

- Utility Power Meters

- GPS

B. NRF

The NRF24101 is a single chip $2.4 \mathrm{GHz}$ transceiver designed for ultra-low power wireless applications. The NRF24L01 is designed for operation in the world-wide ISM frequency band $2.400-2$. 4835GHz.The connections for transmitter and receiver are same. CE and CSN are used to activate the chip. SCK is used to synchronize signals between transmitter and receiver. MISO (master in slave out) and MOSI (master out slave in) are used for data transfer.it gives up to $2 \mathrm{mbps}$ data rate.it has $126 \mathrm{RF}$ channels.it also uses Shock Burst for automatic packet handling, auto acknowledgement and auto retransmit. The NRF uses CRC (cyclic redundancy check) to detect errors. If the NRF receives 2 packets with same identification the it compares the CRC sums from both packets. If the CRC sums are also equal, the last received packet is considered a copy of the previously received packet and discarded by the NRF.

Applications

- $\quad$ Game controllers

- Wireless Peripherals

- $\quad$ Home and commercial automation

- Asset tracking systems 


\section{International Advanced Research Journal in Science, Engineering and Technology}

Vol. 6, Issue 6, June 2019

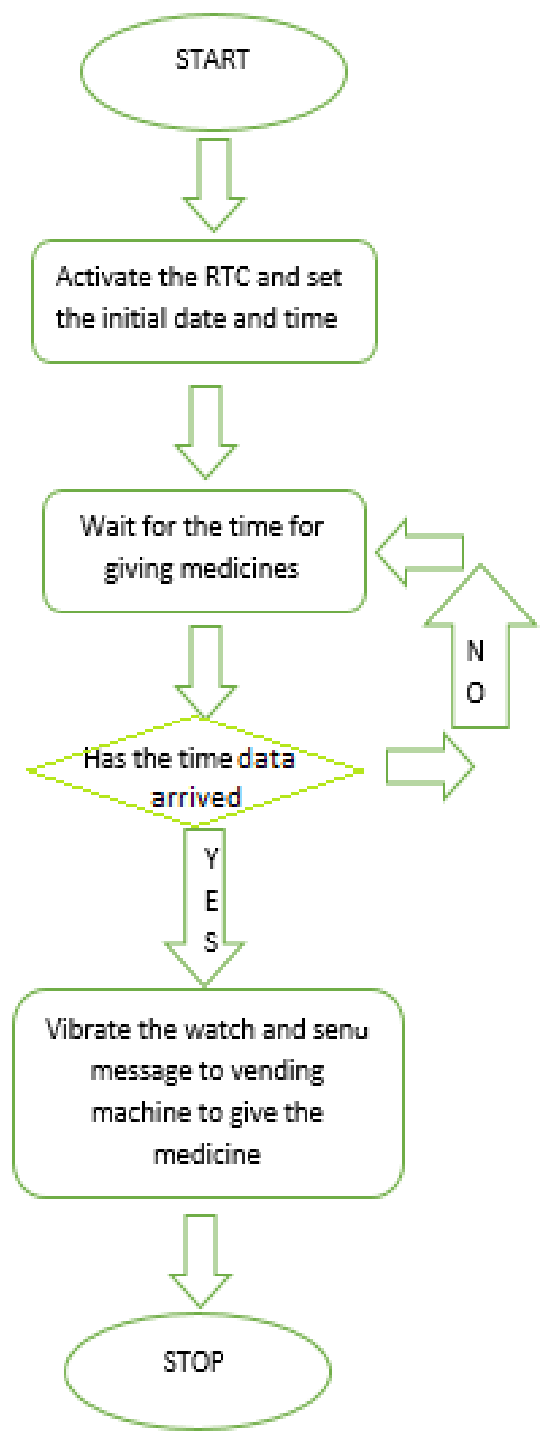

C. Servo (SG90)

The servo motor is an assembly of four things: a normal DC motor, a gear reduction unit, a position-sensing device(potentiometer) and a control circuit. The DC motor is connected with a gear mechanism which provides feedback to a position sensor. As the motor rotates, the potentiometer's resistance changes, so the control circuit can precisely regulate how much movement there is and in which direction. From the gear box, the output of the motor is delivered through servo spline to the servo arm. For standard servo motors, the gear is normally made up of plastic whereas for high power servos, the gear is made up of metal. A servo motor consists of three wires- a black wire connected to ground, a white/yellow wire connected to control unit and a red wire connected to power supply. Servos are controlled by sending an electrical pulse of variable width, or pulse width modulation (PWM), through the control wire. There is a minimum pulse, a maximum pulse, and a repetition rate. A servo motor can usually only turn $90^{\circ}$ in either direction for a total of $180^{\circ}$ movement. The PWM sent to the motor determines position of the shaft, and based on the duration of the pulse sent via the control wire; the rotor will turn to the desired position. The servo motor expects to see a pulse every 20 milliseconds and the length of the pulse will determine how far the motor turns. For example, a pulse width of 1.5 millisecond will turn the motor 90 degrees. A pulse having width less than 1.5 millisecond will move it towards the 0-degree position and pulse having width longer than 1.5 millisecond will turn it towards 180 -degree position.

Applications

- Grippers

- $\quad$ Rudders

- In bipeds

- In Conveyor belts

- In woodworking and CNC 


\section{International Advanced Research Journal in Science, Engineering and Technology}

Vol. 6, Issue 6, June 2019

D. IR sensor

An IR sensor consists of two parts, the emitter circuit and the receiver circuit. This is collectively known as a photocoupler or an optocoupler. The emitter is an IR LED and the detector is an IR photodiode. The IR photodiode is sensitive to the IR light emitted by an IR LED. The photo-diode's resistance and output voltage change in proportion to the IR light received. An Infrared light emitting diode (IR LED) is a special purpose LED emitting infrared rays ranging $700 \mathrm{~nm}$ to $1 \mathrm{~mm}$ wavelength. IR LEDs are usually made of gallium arsenide or aluminium gallium arsenide. The photodiode receives the radiation emitted by the IR LED once reflected back by the object. Closer the object, higher will be the intensity of the incident radiation on the photodiode. This intensity is converted to voltage to determine the distance. The intensity is converted into voltage linearly. A photodiode has certain quantum efficiency. So, for example if a detector has an efficiency of 0.5 amps per watt, and the load resistance is 50 Ohm, then the sensitivity would be given by formula

sensitivity $=$ effeciency $*$ resistance

$\therefore$ sensitivity $(\mathrm{S})=50 * 0.5 \mathrm{~A} * \Omega / \mathrm{W}$ as $\boldsymbol{W}=\boldsymbol{V} * \boldsymbol{A}$ and $\boldsymbol{R}=\frac{\boldsymbol{V}}{\boldsymbol{I}}$

$\mathrm{S}=25 \mathrm{I}-1$

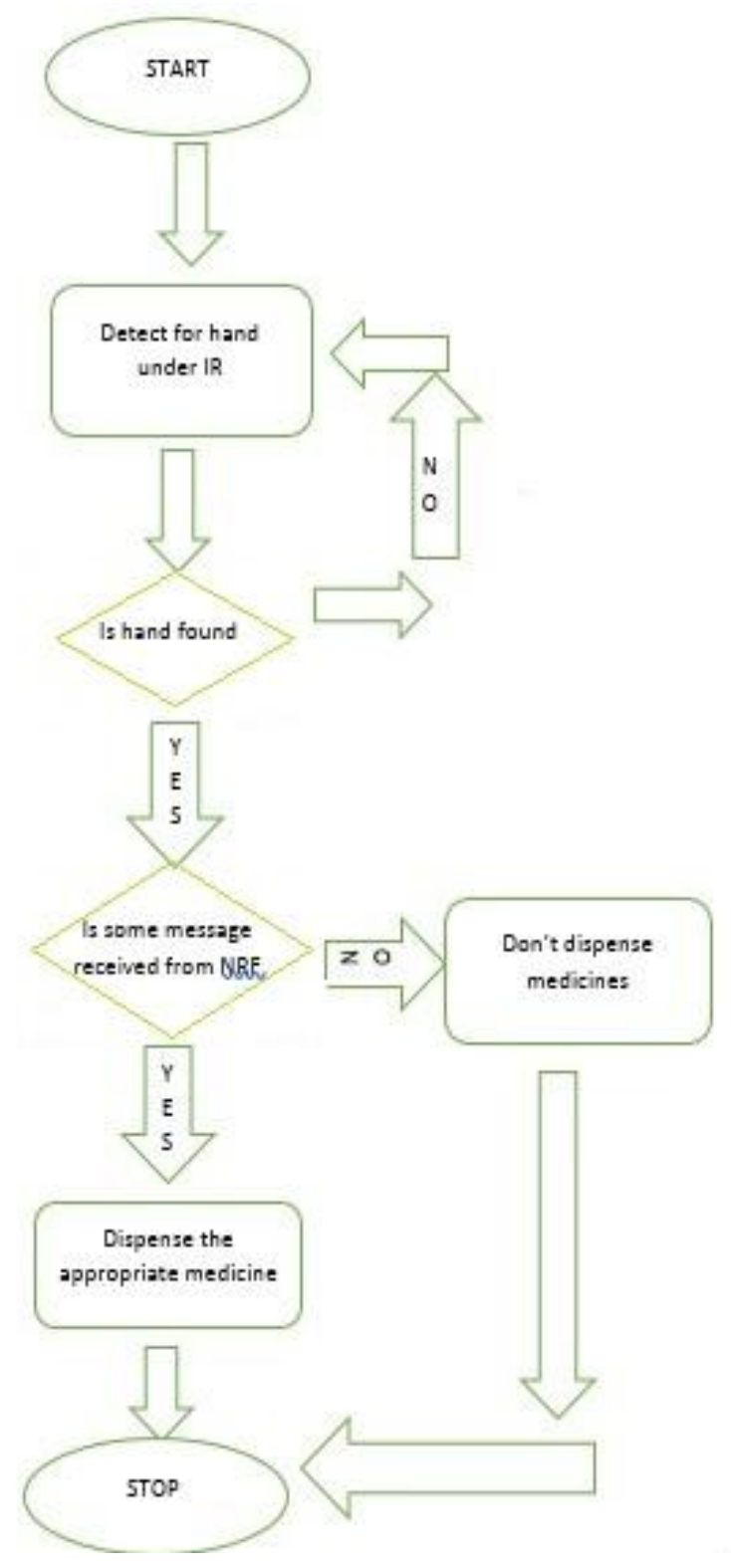

E. IOT

Internet of Things (IoT) is an ecosystem of connected physical objects that are accessible through the internet. That is, objects that have been assigned an IP address and have the ability to collect and transfer data over a network without 


\title{
International Advanced Research Journal in Science, Engineering and Technology
}

\author{
Vol. 6, Issue 6, June 2019
}

manual assistance or intervention. These are some of the technologies support the specific networking functionality needed in an IoT system-

A.NFC and RFID: RFID (radio frequency identification) technology employs 2-way radio transmitter-receivers to identify and track tags associated with objects. NFC (near field communication) consists of communication protocols for electronic devices, typically a mobile device and a standard device.

B. Low-Energy Bluetooth

This technology supports the low-power, long-use need of IoT function while exploiting a standard technology with native support across systems.

\section{Low-Energy Wireless}

This technology replaces the most power consuming aspect of an IoT system. Though sensors and other elements can power down over long periods, communication links must remain in listening mode. Low-energy wireless not only reduces consumption, but also extends the life of the device through less use.

\section{Radio Protocols}

ZigBee, Z-Wave, and Thread are radio protocols for creating low-rate private area networks. These technologies are low-power, but offer high throughput unlike many similar options. This increases the power of small local device networks without the typical costs.

\section{E. LTE-A}

LTE(Long-Term Evolution) is a standard for high speed wireless communication for mobile devices and data terminals, based on the GSM(global system for mobile),EDGE(enhanced data rates for GSM evolution),UMTS(universal mobile telecommunication system),HSPA(high speed packet access) technologies. LTE-A (LTE Advanced) delivers an important upgrade to LTE technology by increasing not only its coverage, but also raising its throughput. It gives IoT a tremendous power through expanding its range

\section{F. Wi-Fi-Direct}

Wi-Fi-Direct eliminates the need for an access point. It allows P2P (peer-to-peer) connections with the speed of Wi-Fi. Wi-Fi-Direct eliminates an element of a network that often bogs it down, and it does not compromise on speed or throughput.

\section{POWER MANAGEMENT}

- Power management means powering the gear.

- Lithium Ion which is used in mobiles can be used in suggested gear.

- Most devices work on 3.3V, so we can have a battery of $4.3 \mathrm{~V}$ or maximum $5 \mathrm{~V}$.

- All devices have to be managed for efficient power utilisation.

- A lithium-ion battery or Li-ion battery is a type of rechargeable battery in which lithium ions move from the negative electrode to the positive electrode during discharge and back when charging.

- Because of the inherent instability of lithium metal, especially during charging, research shifted to a non-metallic lithium battery using lithium ions. Although slightly lower in energy density than lithium metal, lithium-ion is safe

\section{IMPLEMENTATION AND OTHER ISSUES}

- Major issue is balancing high speed, minimum space with least power consumption.

- We have to choose a particular battery with proper current rating so we can manage so much peripherals or interfaces.

- For handling so many peripherals we have to be very particular in programming.

\section{FUTURE MODIFICATIONS}

- The man's schedule will also be maintained by the medicine dispenser through IOT.

- The box will not only maintain his schedule and show him by the band but also give his medicines to him before some time or after some time if his schedule does not permit him to take the medicine at specified time

- We can use ARM microcontroller or Raspberry Pi which will enable it to use internet and give a more stable connection than using node MCU or ESP8266. We can send the alerts through internet too.

- We can also dispense medicines in form of syrups using a pump motor. 


\section{International Advanced Research Journal in Science, Engineering and Technology}

Vol. 6, Issue 6, June 2019

- With some modifications we can also install this device in public places and people can use RFID cards to store information about that person and also to pay for the medicines.

\section{RESULTS}
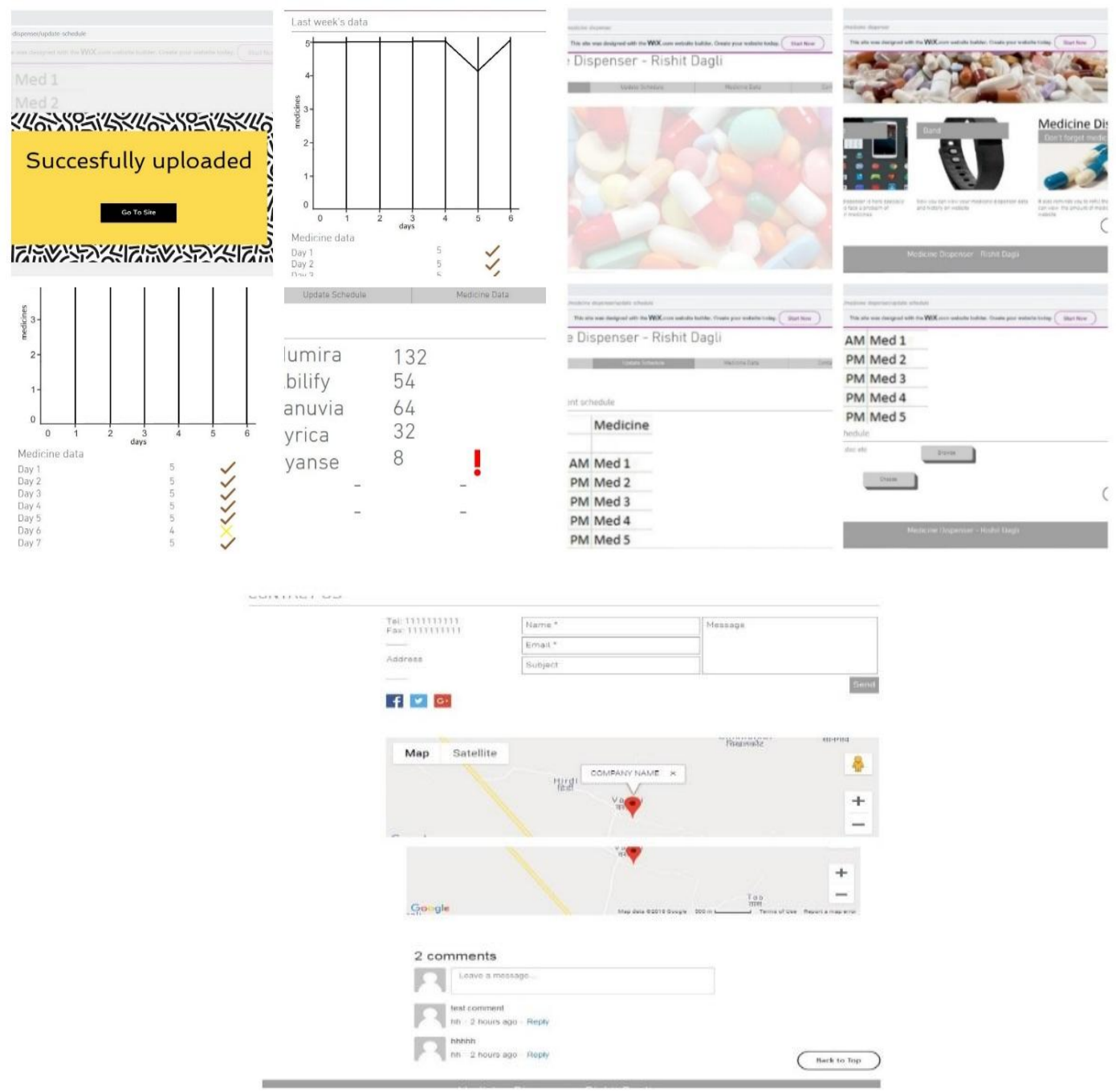

\section{REFERENCES}

[1]. https://datasheets.maximintegrated.com/en/ds/DS3231.pdf

[2]. https://www.sparkfun.com/datasheets/Components/nRF24L0 1_prelim_prod_spec_1_2.pdf

[3]. https://www.espressif.com/sites/default/files/documentation/0a-esp8266ex_datasheet_en.pdf

[4]. https://www.arduino.cc

[5]. https://cdn-learn.adafruit.com/downloads/pdf/ir-sensor.pdf

[6]. http://akizukidenshi.com/download/ds/towerpro/SG90.pdf

[7]. https://learn.adafruit.com/category/learn-arduino

\section{BIOGRAPHY}

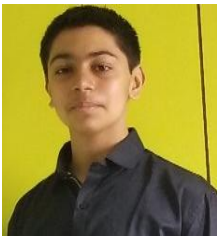

Name: Rishit Dagli

Affiliation: Student, Thakur International School(std-9) Mumbai-400067 India.

Member of Young engineer's club, Science Kidz Mumbai-400067 India

Interest Area: Robotics, app development, Arduino programming, python, raspberry pi, artificial intelligence

Ambition: AI development, making specialised robots for DRDO and society at large 